\title{
Transporting Powder with Surface Acoustic Waves Propagating on Tilted Substrate
}

\author{
Tsunemasa Saiki, ${ }^{1,2 *}$ Yukako Takizawa, ${ }^{1}$ Takahiro Kaneyoshi, ${ }^{1}$ Kenji Iimura, ${ }^{3}$ \\ Michitaka Suzuki, ${ }^{4}$ Akinobu Yamaguchi, ${ }^{5}$ and Yuichi Utsumi ${ }^{5}$ \\ ${ }^{1}$ Hyogo Prefectural Institute of Technology, 3-1-12, Yukihira-cho, Suma-ku, Kobe 654-0037, Japan \\ ${ }^{2}$ Department of Electrical Materials and Engineering, Graduate School of Engineering, University of Hyogo, \\ 2167, Shosha, Himeji 671-2280, Japan \\ ${ }^{3}$ Department of Chemical Engineering, Graduate School of Engineering, University of Hyogo, \\ 2167, Shosha, Himeji 671-2280, Japan \\ ${ }^{4}$ Institute for Research Promotion and Collaboration, University of Hyogo, \\ 3F, Jibasan Building, 123, Minamiekimae-cho, Himeji 670-0962, Japan \\ ${ }^{5}$ Laboratory of Advanced Science and Technology for Industry, University of Hyogo, \\ 3-1-2, Koto, Kamigori-cho, Ako-gun, Hyogo 678-1205, Japan
}

(Received December 9, 2020; accepted February 25, 2021; online published March 8, 2021)

Keywords: surface acoustic wave, actuator, powder transport, piezoelectric material, gravity force

We fabricated a surface acoustic wave (SAW) actuator by patterning an interdigital transducer (IDT) made of $\mathrm{Al}$ film on a $\mathrm{LiNbO}_{3}$ substrate and investigated its transport characteristics with powders of spherical copper, spherical glass, spherical polyethylene, and angular alumina particles with sizes of about $100 \mu \mathrm{m}$. The IDT had a $2 \mathrm{~mm}$ pitch size, $10 \mathrm{~mm}$ aperture, and 10 stripline pairs. It was found that the glass, polyethylene, and alumina powders that could not be transported when the actuator was horizontal could be transported by tilting the actuator, i.e., the powder transport path. It was also found that the powder movement response times with SAWs were shorter than $50 \mathrm{~ms}$ when the actuator, tilted at suitable angles of $0^{\circ}, 6^{\circ}, 15^{\circ}$, and $26^{\circ}$, transported the copper, glass, polyethylene, and alumina powders, respectively. Then, we fabricated miniature feeders by mounting hoppers on SAW actuators tilted at the above angles and investigated their powder feed characteristics. It was found that, when the feeders were driven for $50 \mathrm{~ms}$ at an electric power of $1 \mathrm{~W}$, the fed weights of the copper and glass powders, which did not have flowability problems, were 2.3 and $0.9 \mathrm{mg}$, respectively.

\section{Introduction}

Recently, in the field of life sciences, there has been a need to physically handle tiny amounts of dry micropowders with high accuracy. If such technology can be established, the number of analysis targets for micro total analysis systems ( $\mu$ TAS) will increase because not only liquids but also powders can be used as reagents or samples, ${ }^{(1-3)}$ and moreover, custom-made medicines will be commonly supplied because powder can be automatically fed with accuracy beyond human capacity. However, it is impossible to physically handle such dry powders using a general-purpose conveyor or feeder that has been miniaturized.

*Corresponding author: e-mail: saiki@hyogo-kg.jp

https://doi.org/10.18494/SAM.2021.3213 
An ultrasonic wave device and surface acoustic wave (SAW) actuator for transporting a tiny amount of dry powder have been studied. ${ }^{(4-7)}$ The ultrasonic wave device consists of two parts, a wave-generating source made of a piezoelectric multilayer and a powder transport path made of an acrylic pipe or a substitute for it. The SAW actuator consists of a single part having both a wave-generating source and a powder transport path because an interdigital transducer (IDT) for generating SAWs is fabricated on a piezoelectric substrate whose surface is used as a transport path. Therefore, the SAW actuator has a simpler structure than the ultrasonic wave device and has the major advantage of being easily fabricated. Note that, in related studies on SAW actuators, liquid flow has been mainly reported, ${ }^{(8-14)}$ and there are very few reports on the transport of solids. ${ }^{(7,15)}$

Currently, copper powder has been the only powder reported to be transported toward the SAW downstream side. ${ }^{(7)}$ Transport toward this side can be used in a feeder and has a wider range of applications than that toward the SAW upstream side. However, in the potential applications, it will be necessary to handle powders made of not only conductors such as copper but also insulators (dielectric materials) such as reagents. It is very difficult to transport a dielectric powder using a SAW actuator as well as other devices because of electrostatic adherence on the transport path of these devices. Note that it is more difficult for a dielectric material to discharge static electricity than a conducting material, and thus, the former easily maintains an electrified state.

To solve this problem, we considered two methods, one that eliminates static charge with an ionizer and one that compensates for kinetic energy applied to a powder by means other than SAWs. In particular, when using the force of gravity as kinetic energy, the latter method of compensation is much lower in cost than the former method of static charge elimination because it does not require any special equipment and can be realized by simply tilting the powder transport path, i.e., the SAW actuator. This very simple tilting method has been widely used for vibratory feeders in general.

Thus, in this study, we targeted various kinds of powders and investigated whether they could be transported by a horizontal SAW actuator, i.e., on a horizontal SAW-propagating surface. Then, we found the reason for not being able to transport specific kinds of powders by SAWs. Moreover, we confirmed that, by tilting the SAW actuator, the number of kinds of powders that could be transported by SAWs was increased, and we closely investigated the powder transport characteristics on the tilted SAW-propagating surface. Finally, on the basis of the obtained results, we designed and fabricated SAW miniature feeders by mounting powder storage hoppers on tilted SAW actuators and investigated their powder feed characteristics.

\section{Powder Transport by SAWs on Horizontal Surface}

In this section, we describe an investigation into the kinds of powders that can be transported on a horizontal SAW-propagating surface. Moreover, focusing on the kinds of powders that could not be transported by SAWs, we also explain why the powders could not be transported.

A photograph of a SAW actuator prepared for the powder transport experiments in this paper is shown in Fig. 1. The SAW actuator was proven to be suitable for copper powder 


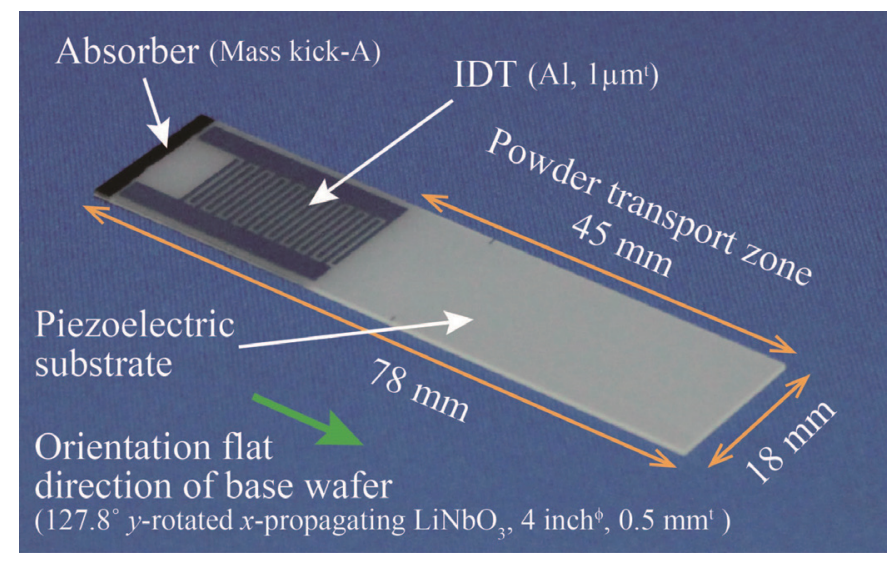

(a)

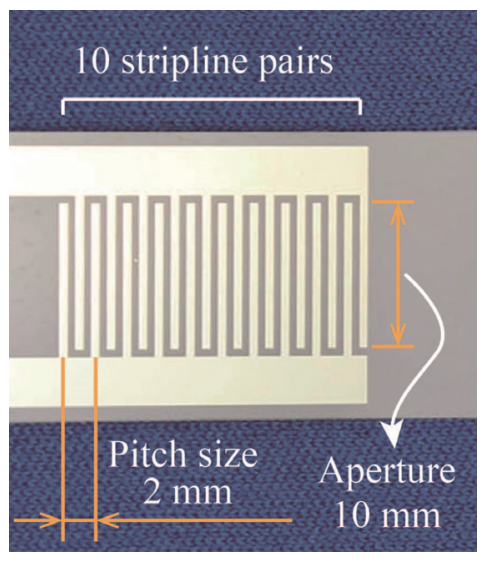

(b)

Fig. 1. (Color online) Photograph of SAW actuator fabricated for powder transport experiment. (a) Overview and (b) magnified view of IDT.

transport in our previous experiment and was fabricated by a single photolithography process performed with semiconductor technology. ${ }^{(7)}$ Concretely, a 4-inch piezoelectric $\left(127.8^{\circ} y\right.$-rotated $x$-propagating $\mathrm{LiNbO}_{3}$ ) wafer was cut to a size of $18 \times 78 \mathrm{~mm}^{2}$ by a dicer (DAD322, Disco Co.). Here, the wafer thickness was $500 \pm 30 \mu \mathrm{m}$ and its surface roughness was $0.3 \mathrm{~nm}$ or less. A $1 \mu \mathrm{m} \mathrm{Al} \mathrm{film} \mathrm{was} \mathrm{formed} \mathrm{on} \mathrm{the} \mathrm{cut} \mathrm{substrate} \mathrm{by} \mathrm{a} \mathrm{DC} \mathrm{sputtering} \mathrm{system} \mathrm{(SDV4320,} \mathrm{Shinko}$ Seiki Co., Ltd.). Then, by using a mask aligner (MA6, SUSS MicroTec, Inc.), UV exposure was applied to a positive resist (OFPR-800, Tokyo Oka Kogyo Co., Ltd.), and an IDT electrode pattern was transferred from a glass mask. After that, by Al etching performed in accordance with this pattern, the IDT was fabricated at a position on one side of the cut substrate. The IDT had a $2 \mathrm{~mm}$ stripline pitch, a $10 \mathrm{~mm}$ aperture, and 10 stripline pairs, and its central axis direction was aligned with the orientation flat direction of the piezoelectric wafer before cutting. In addition, a $45 \times 18 \mathrm{~mm}^{2}$ blank area in front of the IDT was used for transporting powder by SAWs in the experiment. Finally, we hand-printed hot-melt asphaltic adhesive (Mass Kick-A, Furuuchi Chemical Co.) as an absorber to prevent SAW reflection from the edge on one side of the cut substrate. Thus, the SAW actuator was completed.

Optical photomicrographs of the four kinds of powders whose transport characteristics were investigated on the SAW actuator are shown in Fig. 2. Figures 2(a)-2(d) are photographs of copper, glass, polyethylene, and alumina powders, respectively. The copper powder was also used in our past experiment, and we confirmed that it could be transported by SAWs. ${ }^{(7)} \mathrm{We}$ selected the other three kinds of powders because their material properties differed from those of the copper powder (which is a conductor and an inorganic material with spherical particles). Concretely, we chose the glass powder as it functions as an insulator, the polyethylene powder because it is an organic material, and the alumina powder because its particles have an angular shape. The particle sizes of these powders, which were analyzed by a sieve shaker (S-1, Teraoka Co.), are summarized by the cumulative distributions shown in Fig. 3. As can be seen, these powders had the same particle size of about $100 \mu \mathrm{m}$. Concretely, the median (50\%) particle 


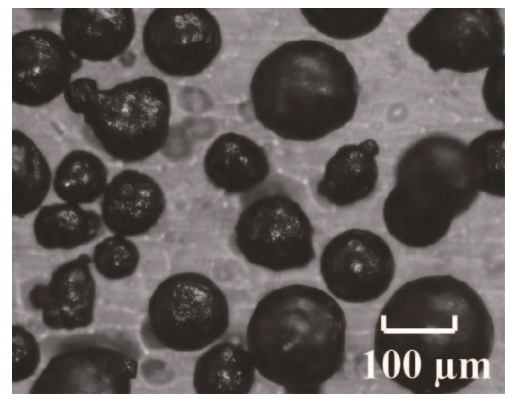

(a)

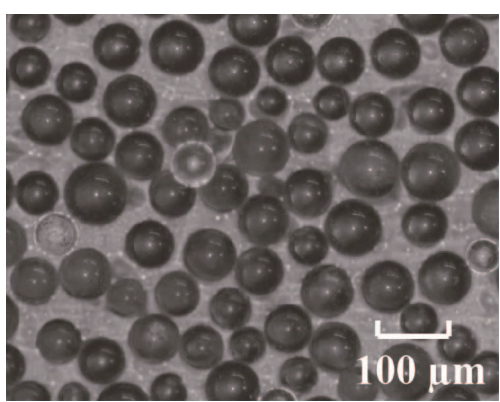

(c)

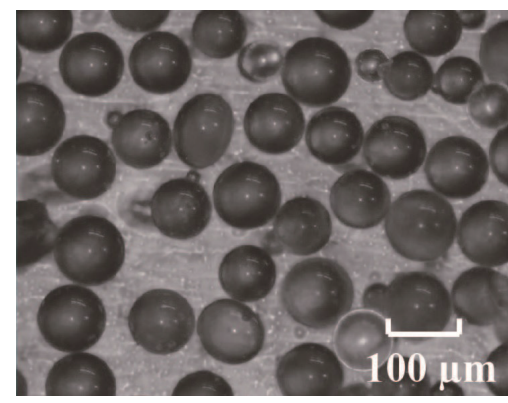

(b)

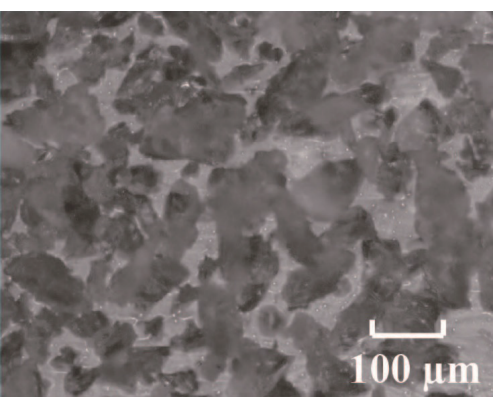

(d)

Fig. 2. Optical photomicrographs of powders used in our experiments. (a) Copper, (b) glass, (c) polyethylene, and (d) alumina.

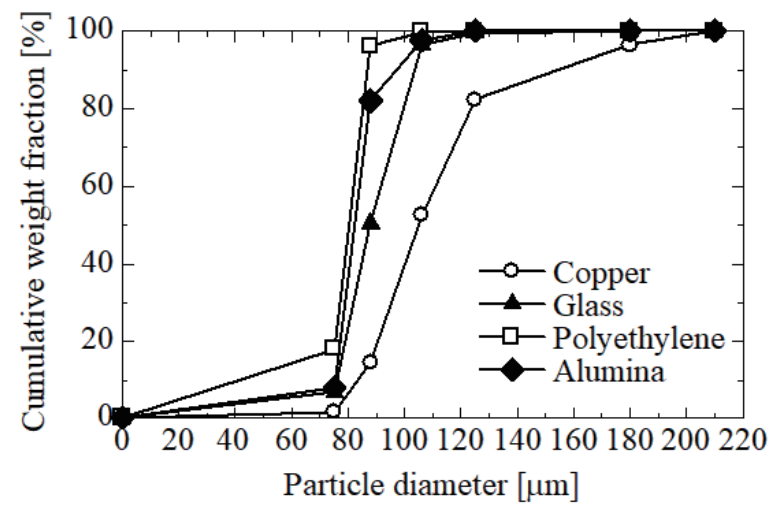

Fig. 3. Particle size distributions of powders used in our experiments.

sizes of the copper, glass, polyethylene, and alumina powders were 104.8, 87.9, 80.2, and $82.3 \mu \mathrm{m}$, respectively. Note that in another experiment, in which the powders were placed on a $\mathrm{LiNbO}_{3}$ wafer, it was found that the repose angles of the copper, glass, polyethylene, and alumina powders were $31^{\circ}, 33^{\circ}, 31^{\circ}$, and $45^{\circ}$ and their sliding angles were $6^{\circ}, 17^{\circ},>90^{\circ}$, and $>90^{\circ}$, respectively. These angles were measured in an environment controlled at a temperature of $22 \pm 2{ }^{\circ} \mathrm{C}$ and humidity of $55 \pm 5 \%$. All experiments described in this paper were carried out under the same conditions. 
Our experimental setup for observing powder transport by SAWs is shown in Fig. 4. Before the experiments, we adjusted the angle of the tilting table, consisting of a goniometer stage (GOH40A35, Sigumakoki Co., Ltd.) and optical baseplates, fixed on a SAW actuator, and then kept the SAW actuator horizontal (a tilting angle $\theta$ of $0^{\circ}$ ). Then, by using a container (a cylindrical shape with $3 \mathrm{~mm}$ diameter and $0.6 \mathrm{~mm}$ depth), we accurately placed $4 \mathrm{~mm}^{3}$ of powder $10 \mathrm{~mm}$ from the IDT.

In the experiments, a function generator (AFG3252, Tektronix Co.) created $1 \mathrm{kHz}$ burst waveforms consisting of 2000 cycles of $3.2 \mathrm{MHz}$ sine waves. From past experience, we knew that, by using these burst waveforms, copper powder can be efficiently transported toward the SAW downstream side. ${ }^{(7)}$ Then, an RF amplifier (ALM000110-2840FM, R\&K Co., Ltd.) boosted the burst waveform voltage. When the toggle switch of the actuator was manually turned on, the boosted voltage was applied from a terminating resistor (50T-116, JFW Industries, Inc.) to an IDT by a coaxial switch (AV204, Stack Electronics Co., Ltd.) controlled through a microcomputer (Arduino Uno Rev.3). At the same time, a light-emitting diode (LED) connected to the microcomputer lit up. Then, by applying the boosted voltage to the IDT, SAWs were generated. Here, we adjusted the voltage amplitude of the burst waveform by using the function generator beforehand so that an electric power of $1 \mathrm{~W}$ could be applied to the IDT. The applied electric power $P_{A}\left(=\right.$ incident power $P_{I}$ - reflected power $\left.P_{R}\right)$ was measured by using a power meter (NRP-2, NRP-Z91, Rohde \& Schwarz, and DC3001M1, AR Co.). Note that the other powder transport experiments described in the following sections were carried out with the same applied electric power of $1 \mathrm{~W}$.

After that, SAWs propagating on the piezoelectric substrate reached and acted on the powder. At this time, we recorded powder movement behaviors at a frame resolution of $1920 \times 1080$ and a frame rate of $60 \mathrm{fps}$ with a high-definition video camera (HDR-CX430V, Sony Co.). After the experiments, we used images of the recorded video to check for the presence or absence of powder transport by SAWs. Moreover, when the powder was transported, we obtained the powder transport speed from the video images and then calculated the powder transport efficiency by dividing the powder transport speed by the applied electric power $(1 \mathrm{~W})$. Moreover, since not only the powder movement behavior but also the LED light was recorded in the images, we could also accurately determine the time that the SAW actuator started to be driven.

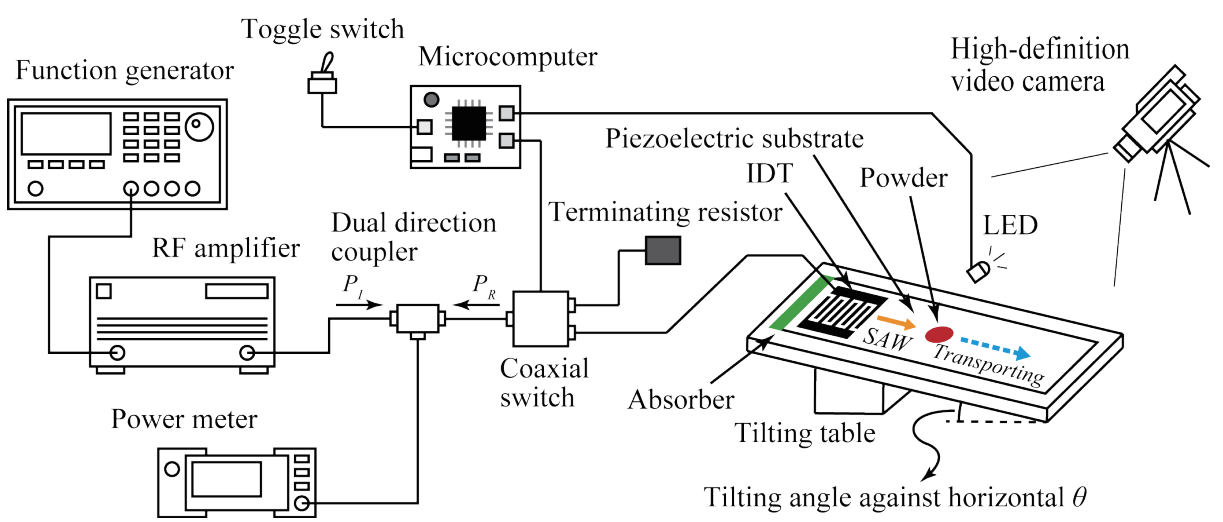

Fig. 4. (Color online) Experimental setup for observing SAW powder transport. 
Photographs of the sequential movement of various powders transported by SAWs are shown in Fig. 5. The photographs in Figs. 5(a)-5(d) were taken when the SAWs were applied to the copper, glass, polyethylene, and alumina powders, respectively. Here, the start time for applying the electric power to the IDT, i.e., driving the SAW actuator, was defined as $t=0 \mathrm{~s}$. As shown in Fig. 5(a), the copper powder was transported further toward the SAW downstream side with elapsed time $t$. The copper powder transport efficiency obtained from the video images of these sequential photographs was $15.4 \mathrm{~mm} /(\mathrm{s} \cdot \mathrm{W})$. More specifically, this value was obtained on the basis of the powder transport speed calculated using the time in which the tip of the powder moved $10 \mathrm{~mm}$ toward the SAW downstream side [Fig. 5(a)]. From Figs. 5(b)-5(d), we confirmed that the glass, polyethylene, and alumina powders were made to diffuse on the piezoelectric substrate surface by the SAWs. However, it is difficult to objectively refer to such diffusion as powder transport because the tips of these powders could not be moved $10 \mathrm{~mm}$ toward the SAW downstream side in $1 \mathrm{~min}(t=60 \mathrm{~s})$.

We thus observed that diffused glass, polyethylene, and alumina powders could not be transported by the SAWs. As seen in Fig. 6(a), in the photograph of glass powder diffusion at $t=20 \mathrm{~s}$ after the SAW actuator started, the glass powder mainly diffused toward the SAW downstream side from the initial position at $t=0 \mathrm{~s}$, and the powder distribution pattern of periodic stripes perpendicular to the SAW propagation direction was caused by the standing waves of the SAWs. Next, as shown in Fig. 6(b), from magnified sequential photographs taken at intervals of $0.5 \mathrm{~s}$, we confirmed that powder particles in the narrow regions of the standing wave antinode, where particle densities are relatively low, moved quickly, and those in the narrow regions of the standing wave node, where particle densities are relatively high, moved slowly. This tendency was also seen in the experiments in which the polyethylene and alumina powders were used. These observations indicate the possibility that a SAW can release powder particles from binding forces generated by static electricity, friction, etc., after which the powder

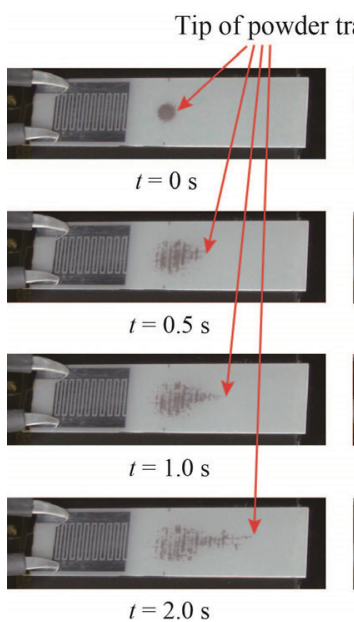

(a)

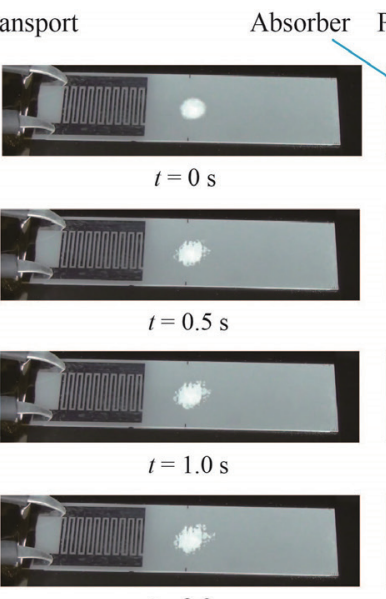

(b)

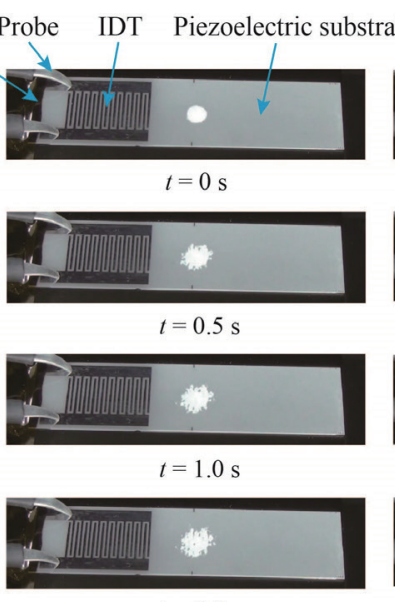

(c)

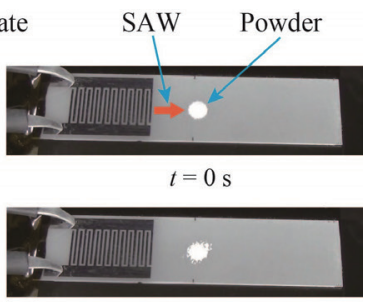

$t=0.5 \mathrm{~s}$

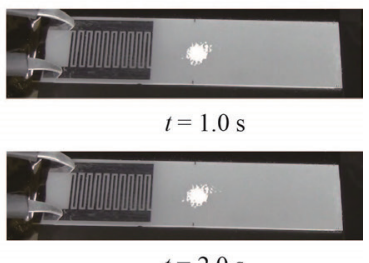

(d)

Fig. 5. (Color online) Examples of sequential movement behavior of various powders to which SAWs were applied $\left(\theta=0^{\circ}, P_{A}=1 \mathrm{~W}\right)$. (a) Copper, (b) glass, (c) polyethylene, and (d) alumina. 


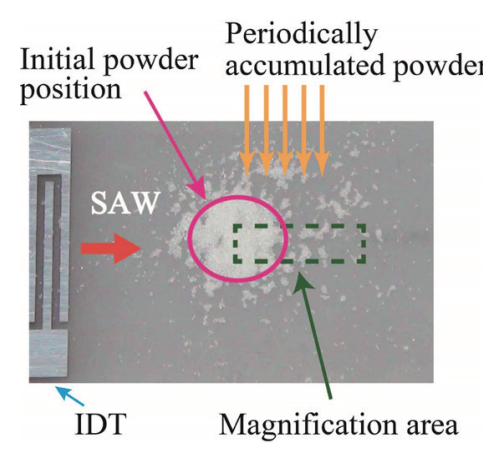

(a)

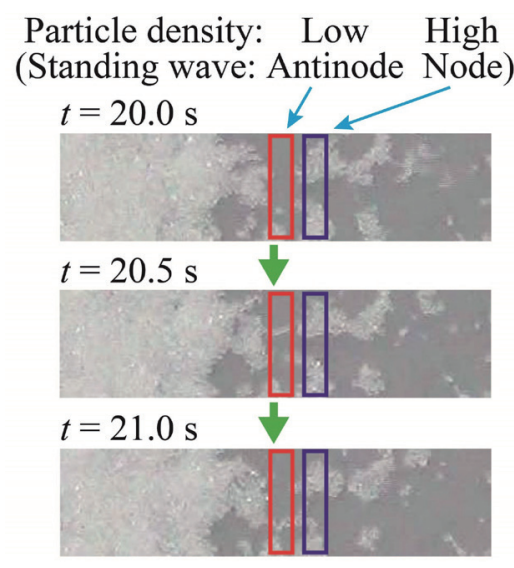

(b)

Fig. 6. (Color online) Glass powder diffused by SAWs $\left(\theta=0^{\circ}, P_{A}=1 \mathrm{~W}\right)$. (a) Overall $(t=20.0 \mathrm{~s})$ and (b) magnification.

particles can move freely regardless of whether there is a standing wave node or antinode region on a SAW-propagating surface.

On the piezoelectric substrate surface on which SAWs did not propagate, the glass powder particles were greatly restrained by static electricity and friction. Therefore, when the piezoelectric substrate surface was tilted $15^{\circ}$ against the horizontal plane, no glass particles moved on the piezoelectric substrate surface. This phenomenon was observed for not only the glass powder but also the polyethylene and alumina powders that could not be transported by the SAWs. These findings show that the powder particles can be released and restrained by the presence and absence of SAW propagation on a powder transport path, respectively. We thus speculated that, if something other than SAWs could uniformly apply a unidirectional force to all the powder particles, the starting and stopping of powder particle transport, even for the powders that could not be transported by only SAWs, could be controlled by the presence and absence of SAWs, respectively.

\section{Powder Transport by SAWs on Tilted Surface}

From the results of the previous section, we learned that, by applying not only the force generated by SAWs but also other forces to powder particles, there is the possibility of transporting powders that could not be transported by SAWs alone. Thus, as another force, we focused on gravity, which can be easily used without special equipment. In this section, we demonstrate an increase in the number of powder types that can be transported when gravity is uniformly applied to all particles by tilting a powder transport path on which a SAW propagates, in simple words, a SAW actuator. Moreover, we describe the powder transport characteristics.

Examples of sequential photographs of powder transport by SAWs on a tilted surface are shown in Fig. 7. The photographs in Figs. 7(a)-7(c) were taken when glass, polyethylene, and alumina powders were transported on tilted surfaces at tilting angles $\theta$ of $10^{\circ}, 20^{\circ}$, and $30^{\circ}$, respectively. From these figures, we found that the powders that could not be transported in a 


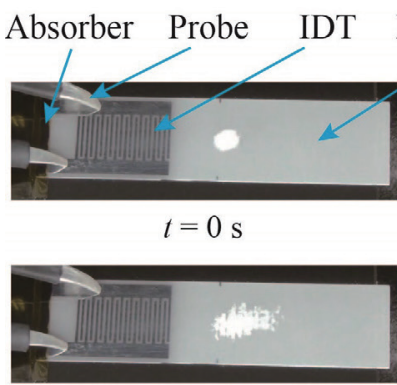

$t=0.5 \mathrm{~s}$

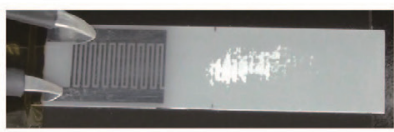

$t=1.0 \mathrm{~s}$

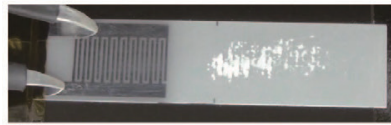

$t=2.0 \mathrm{~s}$

(a)

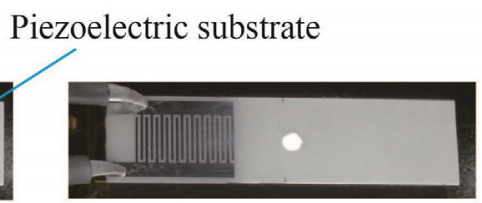

$t=0 \mathrm{~s}$

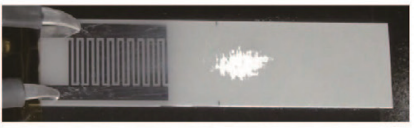

$t=0.5 \mathrm{~s}$

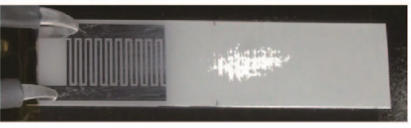

$t=1.0 \mathrm{~s}$

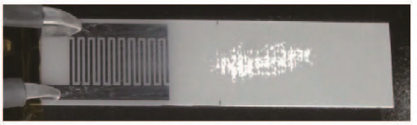

$t=2.0 \mathrm{~s}$

(b)

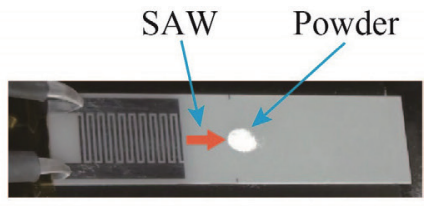

$t=0 \mathrm{~s}$

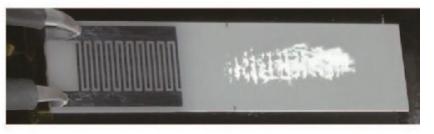

$t=0.5 \mathrm{~s}$

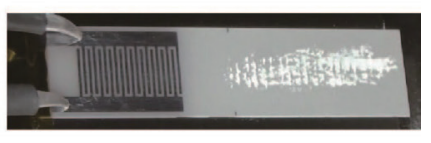

$t=1.0 \mathrm{~s}$

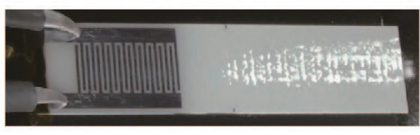

$t=2.0 \mathrm{~s}$

(c)

Fig. 7. (Color online) Sequential photographs of powder transport by SAWs on tilted surface $\left(P_{A}=1 \mathrm{~W}\right)$. (a) Glass, $\theta=10^{\circ}$, (b) polyethylene, $\theta=20^{\circ}$, and (c) alumina, $\theta=30^{\circ}$.

horizontal state $\left(\theta=0^{\circ}\right)$ could be transported by tilting the powder transport path, i.e., increasing $\theta$. The transport efficiencies of the glass, polyethylene, and alumina powders obtained by analyzing the recorded video images were $16.5,18.3$, and $41.2 \mathrm{~mm} /(\mathrm{s} \cdot \mathrm{W})$, respectively. These values are greater than or equal to that of the copper powder at $\theta=0^{\circ}$. Thus, by using the force of gravity as a supplement, i.e., by simply tilting the SAW actuator, more types of powders can be transported by SAWs.

Next, the characteristics of the dependence of powder transport efficiency on the tilting angle are shown in Fig. 8. The horizontal and vertical axes represent the tilting angle $\theta$ and the powder transport efficiency $E$, respectively. Here, we measured the powder transport efficiency in a tilting angle range of $0^{\circ}$ to $30^{\circ}$ (the maximum tilting angle of the tilting table) at intervals of $5^{\circ}$. Note that, if the sliding angle of the powder was less than $30^{\circ}$, we measured the powder transport efficiency in the range from $0^{\circ}$ to its sliding angle. The white circles, triangles, squares, and diamonds represent raw data for copper, glass, polyethylene, and alumina powders, respectively. The gray symbols represent average values calculated from the raw data. It was found that, for the copper, glass, and polyethylene powders, the average transport efficiency increased as the tilting angle increased. In comparison, it was found that the alumina powder could not be transported at tilting angles smaller than $20^{\circ}$, and at the largest tilting angles $\left(20^{\circ}\right.$ to $30^{\circ}$ ), the powder transport efficiency rapidly increased as the tilting angle increased.

Here, we discuss the tilting angle most suitable for transporting each kind of powder. When considering only the powder transport efficiency, the most suitable tilting angle would be slightly smaller than the sliding angle of each kind of powder because of its maximum powder transport efficiency. However, when choosing such an angle as the tilting angle of the SAW 


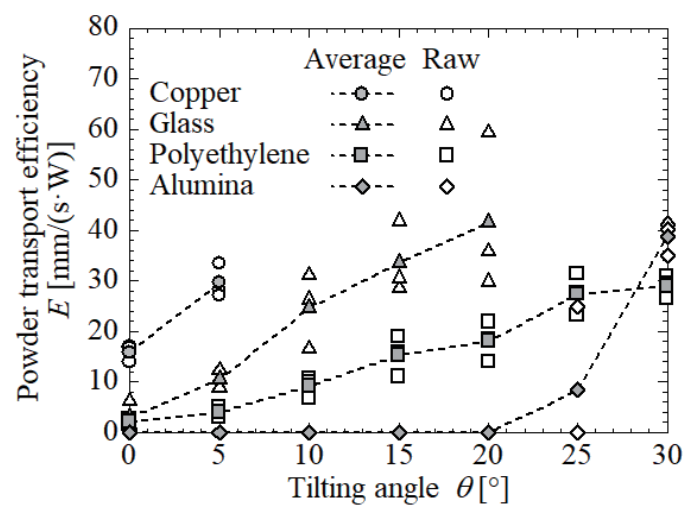

Fig. 8. Dependence characteristics of powder transport efficiency on tilting angle.

actuator, it is difficult to accurately control powder transport by SAWs because the powder is very strongly influenced by the force of gravity. For example, we can easily imagine that, when the SAW actuator is stopped, the powder does not immediately stop but continues to roll down the substrate.

Therefore, since from our previous experiment, we knew that copper powder could be transported with high accuracy by the SAW actuator, ${ }^{(7)}$ we focused on the average powder transport efficiency of copper at $\theta=0^{\circ}$ of $15.4 \mathrm{~mm} /(\mathrm{s} \cdot \mathrm{W})$ obtained from this experiment. We then calculated the tilting angle of each kind of powder giving the same average powder transport efficiency by using the least-squares fitted line drawn by using average values. The calculated tilting angles of the glass, polyethylene, and alumina powders were $6^{\circ}, 15^{\circ}$, and $26^{\circ}$, respectively. In this study, we temporarily defined these calculated tilting angles as the tilting angles suitable for transporting the powders. Note that when the suitable tilting angle of $0^{\circ}$ for copper powder was also included with the suitable tilting angles of the other powders, the tilting angle suitable for transporting powder by the SAW actuator tended to increase with the sliding angle of the powder (see Sect. 2, 3rd paragraph).

\section{Response Time of Powder Particles Moved by SAWs}

Using the method mentioned in the previous section, we decided the SAW actuator's tilting angles suitable for transporting the four kinds of powders. In this section, to verify the validity of our tilting angles, we describe the particle movement response time when using SAWs.

To investigate the response times, we modified the program of the microcomputer drawn in Fig. 4. With this modification, when we turned on the toggle switch, the SAW actuator could be driven once for $1 \mathrm{~s}$ and then five times for $0.5 \mathrm{~s}$, as shown in Fig. 9. At $1 \mathrm{~s}$, the powder stored by the cylindrical container mentioned in Sect. 2 was scattered, and most of the powder particles were in direct contact with the surface on which the SAWs propagated. When the actuator was subsequently driven five times for $0.5 \mathrm{~s}$, we measured two different movement response times for the powder particles. (This measurement was performed by visual observation on the basis of a series of frame images recorded by a high-definition video camera.) One was the interval from starting the SAW actuator to any of the powder particles moving, and the other was that 


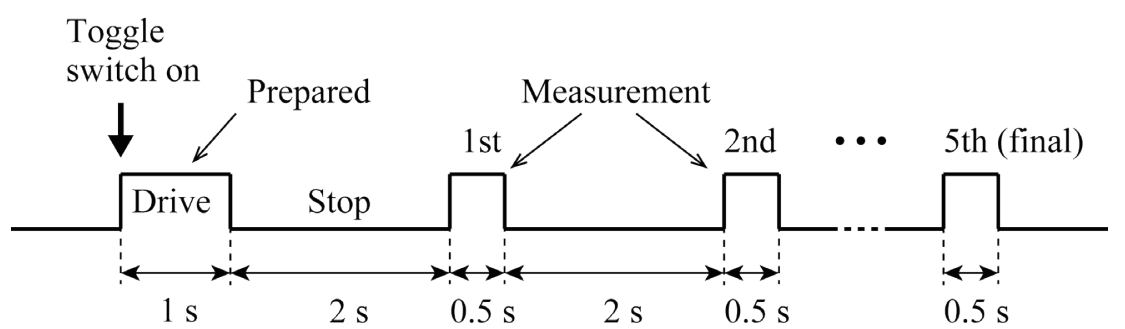

Fig. 9. Timing chart of SAW actuator operation when investigating movement response times of powder particles.

from stopping the SAW actuator to stopping all of the powder particles. Hereinafter, the two times are referred to as the response time until the powder moved and the response time until the powder stopped.

The two response times of the powder movements had time widths because they were obtained from video images recorded at a frame rate of $60 \mathrm{fps}$ with the high-definition video camera drawn in Fig. 4. Here, we concretely consider the response time until the powder moved when the LED light was turned on at the 0th frame and then the powder moved at the $i$ th frame (Fig. 10). In our experimental system, the switching time of the coaxial switch from the terminating resistor side to the IDT side was $2.9 \mathrm{~ms}$, which was an actual value measured by an oscilloscope. Therefore, the minimum and maximum response times until the powder moved were $2.9+16.7 \times i$ [ms] (Case 1) and $13.8+16.7 \times i[\mathrm{~ms}]$ (Case 2), respectively. In the same manner, in consideration of the $3.5 \mathrm{~ms}$ switching time of the coaxial switch from the IDT side to the terminating resistor side, the minimum and maximum response times until the powder stopped were $-3.5+16.7 \times i[\mathrm{~ms}]$ and $13.2+16.7 \times i[\mathrm{~ms}]$, respectively. Note that because the turn-on/off (off to on and on to off) times of the LED were extremely short (100 ns or less), the above-mentioned equations did not take these LED times into account.

The response times until the powder moved and stopped, obtained from the video images, are summarized in Figs. 11(a) and 11(b), respectively. As shown in Fig. 11(a), the response time until the powders moved did not depend on the combination of the powder material and the tilting angle $\theta$ of the SAW actuator, and the powders began to move 14 to $30 \mathrm{~ms}$ after the SAW actuator was started. As shown in Fig. 11(b), the response times until the stopping of the copper powder at $\theta=0^{\circ}$, the glass powder at $\theta=6^{\circ}$, the polyethylene powder at $\theta=15^{\circ}$, and the alumina powder at $\theta=26^{\circ}$ were 26 to $30 \mathrm{~ms}, 30$ to $47 \mathrm{~ms}, 10$ to $27 \mathrm{~ms}$, and 0 to $13.2 \mathrm{~ms}$, respectively; thus, the response time until the powder stopped depended on the combination of the powder material and the tilting angle of the SAW actuator. It is particularly noteworthy that the alumina powder at the largest tilting angle could be stopped in the shortest time among the four kinds of prepared powders. From this finding, it was confirmed that the movement behavior of minute particles is more affected by their shape or material properties, e.g., electrostatic property, than by the force of gravity.

The above results clarify that, for all combinations of powders and tilting angles, the response time until the powder either moved or stopped was shorter than 1/20 s. This indicates that the SAW actuator had the potential to transport powder with a good response time, and the validity of our suitable tilting angles for transporting powders was verified. 


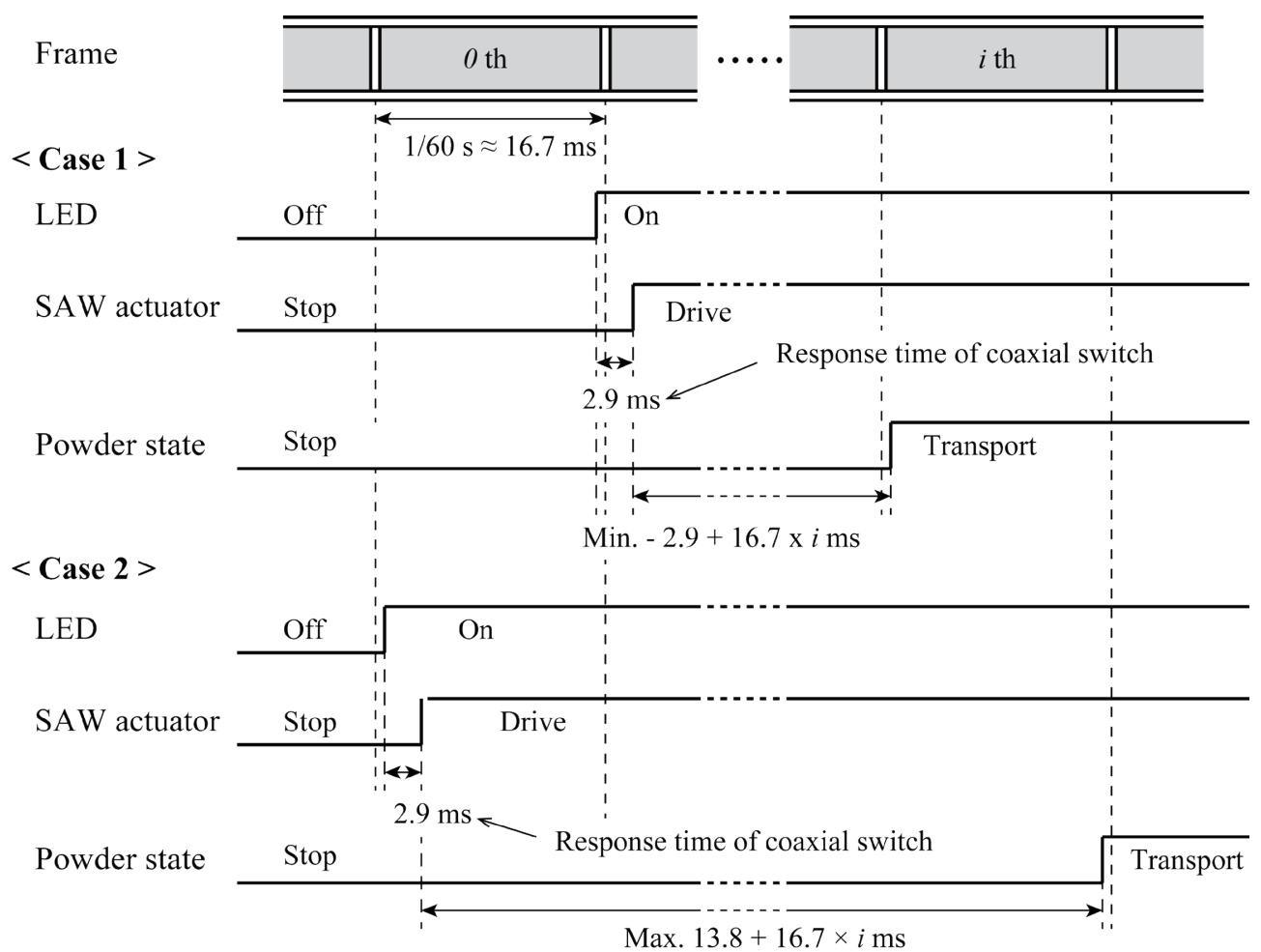

Fig. 10. Method for obtaining response times of powder movement from video images recorded when driving SAW actuator.

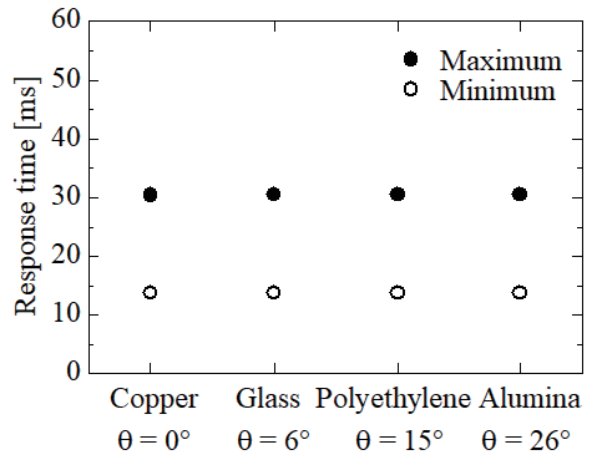

(a)

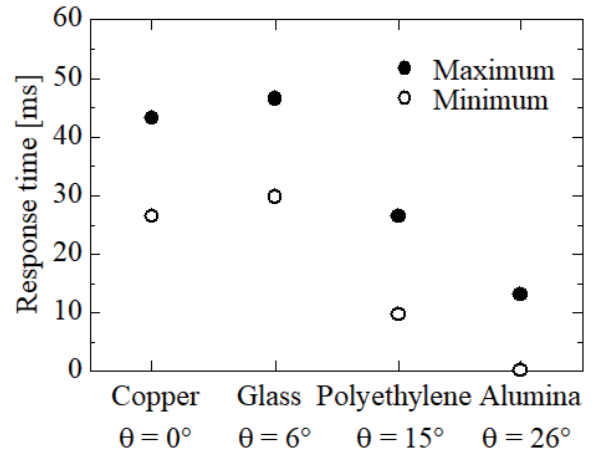

(b)

Fig. 11. Response times of powder movements induced by SAW actuator $\left(P_{A}=1 \mathrm{~W}\right)$. (a) When moving powder and (b) when stopping powder.

\section{Powder Feed Characteristics of SAW Feeders}

As indicated by the experimental results in Sects. 2 and 3, by tilting the SAW actuator from the horizontal state, the range of powders that could be transported increased. Then, as indicated by the experimental results in Sect. 4, the response times of the SAW actuator were short when it was tilted at a suitable angle. In this section, we describe the powder feed characteristics of 
SAW feeders fabricated by mounting a powder storage hopper on an actuator tilted at a suitable angle.

A photograph of four miniature feeders driven by SAWs is shown in Fig. 12. The feeders were fabricated by fixing powder storage hoppers and guide walls on SAW actuators, explained in Sect. 2, with double-sided tape (NICETACK NW-50, Nichiban Co., Ltd.). The powder storage hoppers and guide walls were a single part that was fabricated by using a 3D printer (Connex 500, Stratasys, Inc.) and was made of hard resin (VeroWhite Plus).

The SAW feeders used for transporting copper, glass, polyethylene, and alumina powders are shown from left to right in Fig. 12. In the experiments, the feeders were tilted at the suitable angles mentioned in Sect. 3. Therefore, to keep the upper plane of the hopper horizontal, the upper plane of the hopper and the lower plane of the guide walls, i.e., the plane of the powder transport path, were not parallel (see Fig. 13). Note that the angle $\theta$ made by these planes corresponded to the suitable angle. The hopper capacity was $0.4 \mathrm{~cm}^{3}$, and its shape was an inverted cone with an apex angle of $60^{\circ}$ and height of $10 \mathrm{~mm}$. The hopper had a hole at its bottom for supplying powder to the transport path of the SAW actuator. The hole diameter was $1 \mathrm{~mm}$ for the copper and glass powders and $1.5 \mathrm{~mm}$ for the polyethylene and alumina powders. The apex angle and hole diameter of the hopper were decided in consideration of the powder flowability when using only the hopper without the SAW actuator.

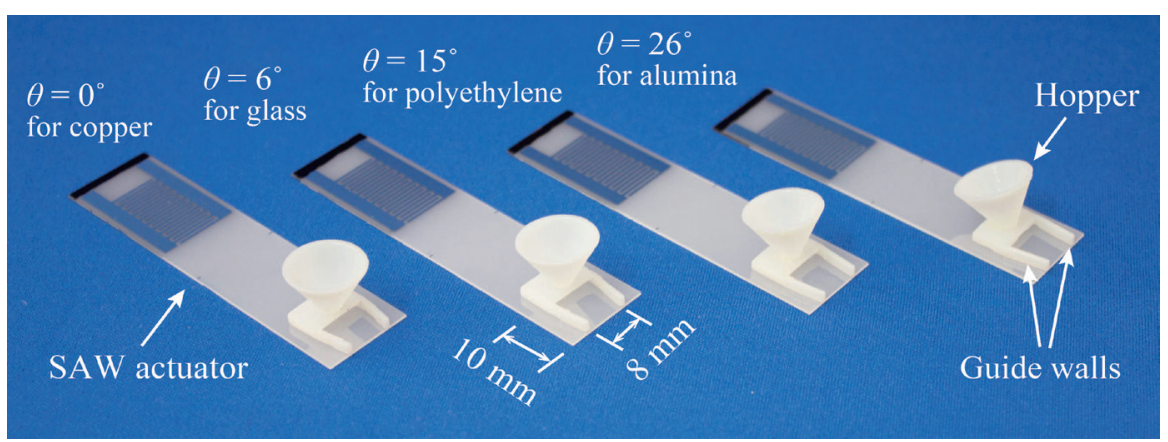

Fig. 12. (Color online) Fabricated SAW feeders.

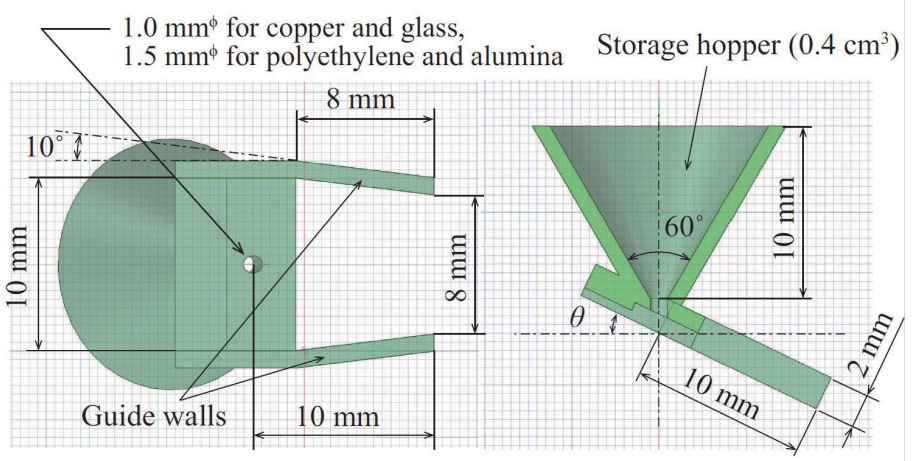

(a)

(b)

Fig. 13. (Color online) Design of hopper and guide walls. (a) View from below and (b) cross-sectional view. 
The guide walls fixed on the SAW actuator had a height of $2 \mathrm{~mm}$ and a length of $10 \mathrm{~mm}$. They were located at a convergent angle of $20^{\circ}$ against an outlet with $8 \mathrm{~mm}$ width. Thus, by employing the short guide walls and fixing them on the substrate edge, we shortened the powder transport path and reduced the amount of transported powder because we expected that the total inertia force of the transported powder would be greatly reduced and thereby the powder feed responsiveness with SAWs would be improved. Note that if the short guide walls are fixed on the IDT side, we can cut most (about three-quarters) of the powder transport zone of the SAW actuator and thereby fabricate a feeder half the size of the feeders shown in Fig. 12. However, in this study, we fabricated the SAW feeder on the basis of a SAW actuator that was not cut because we already knew its powder transport characteristics and drive frequency, as described in the previous sections.

The experimental setup for investigating powder feed characteristics with our fabricated SAW feeders is shown in Fig. 14. Before this experiment, we tilted the table on which the SAW feeder was mounted at the suitable angle for transporting each kind of powder $\left(0^{\circ}\right.$ for copper, $6^{\circ}$ for glass, $15^{\circ}$ for polyethylene, and $26^{\circ}$ for alumina) and then placed $80 \%$ of the maximum powder volume (2100 mg of copper, $500 \mathrm{mg}$ of glass, $200 \mathrm{mg}$ of polyethylene, and $600 \mathrm{mg}$ of alumina) in the hopper. Next, by manually turning the toggle switch on, high-frequency electric power was applied from the terminating resistor to the IDT by the coaxial switch controlled through the microcomputer, and the SAW feeder was then started. Here, the start time of the SAW feeder was defined as $t=0 \mathrm{~s}$. After that, powder automatically supplied from the hopper by gravity was transported between the guide walls, and the transported powder arrived at the outlet and fell down from the feeder through the outlet. The weight of the fallen powder, $m$, was measured by an electronic balance located under the outlet.

A photograph taken when the SAW feeder $\left(\theta=6^{\circ}\right)$ was feeding glass powder is shown in Fig. 15. It was taken at a shutter speed of $1 / 60 \mathrm{~s}$ at $20 \mathrm{~s}$ after the SAW feeder was started. It can be seen that the powder continuously fell down like a waterfall from the output of the SAW feeder. Thereby, it appears that our fabricated SAW feeder can feed powder uniformly.

The temporal change in the weight of each powder fed from the SAW feeder is shown in Fig. 16. It was found that, for the copper, glass, and polyethylene powders, the SAW feeder began

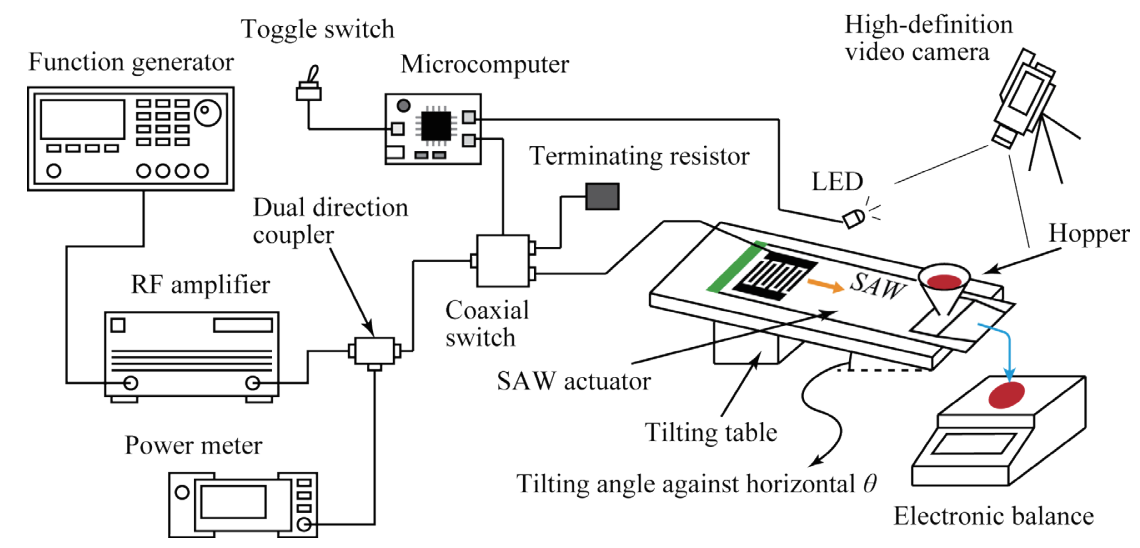

Fig. 14. (Color online) Experimental setup for investigating powder feed characteristics of fabricated SAW feeder. 


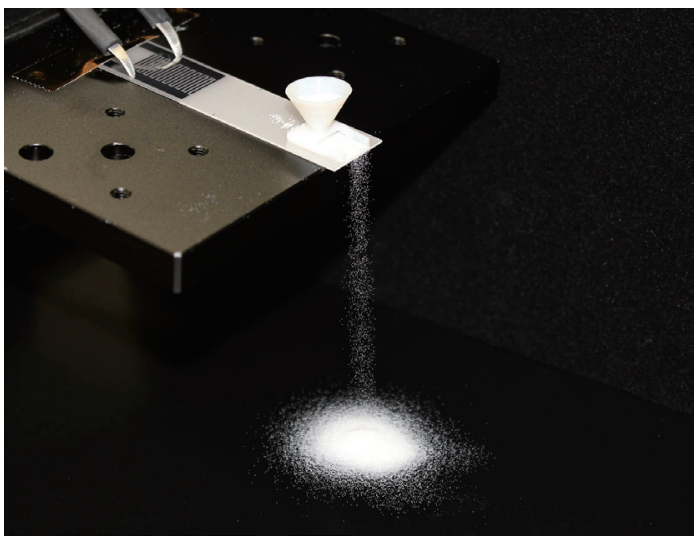

Fig. 15. (Color online) Photograph taken when SAW feeder was feeding glass powder $\left(\theta=6^{\circ}, t=20 \mathrm{~s}\right.$, $\left.P_{A}=1 \mathrm{~W}\right)$.

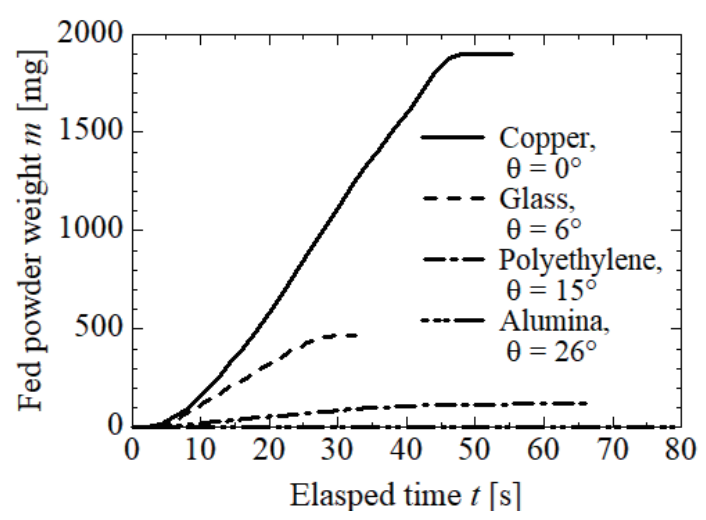

Fig. 16. Temporal change in weight of each kind of powder fed from SAW feeder $\left(P_{A}=1 \mathrm{~W}\right)$.

to feed the powder at about $t=3 \mathrm{~s}$ after starting, and the weight of powder fed from the SAW feeder increased gradually with elapsed time $t$. Moreover, the weights of the copper and glass powders that were fed reached about $90 \%$ of the initial storage weight in the hopper after about 45 and $30 \mathrm{~s}$, respectively. However, the weight of the polyethylene powder did not reach $60 \%$ of the total weight after $1 \mathrm{~min}$. This phenomenon was caused by a rat hole formed in the hopper. ${ }^{(16)}$ Concretely, owing to the formation of the rat hole, the amount of polyethylene powder supplied from the hopper to the powder transport path was limited. Such a problem of powder flowability due to this phenomenon was serious, especially when feeding the alumina powder. Concretely, before driving the SAW feeder, a bank of alumina powder formed under the hopper and clogged the hole of the hopper. Therefore, the alumina powder could not be supplied from the hopper to the powder transport path; thus, no alumina powder could be fed from the SAW feeder. These findings indicate the importance of considering flowability problems, e.g., how to smoothly eject powder from the hopper and how to diffuse the ejected powder on the SAW-propagating surface, when we design a SAW feeder.

Here, we discuss the capabilities of the three SAW feeders that could feed the copper, glass, and polyethylene powders. As shown in Fig. 16, at 10-90\% of the final weight of powder fed from the SAW feeder, the fed powder weight $m$ almost linearly increased with elapsed time $t$. Thus, to determine the feeder capability, we calculated the fed powder weight per unit time from the slope of the straight line determined by the method of least squares for this time period (10-90\% of the final fed powder weight). It was found that the weights per unit time for copper, glass, and polyethylene were 50.1, 20.7, and $3.1 \mathrm{mg} / \mathrm{s}$, respectively, and for all three powders, the correlation coefficient between the fed powder weight and the elapsed time was over 0.997. It was clear from these coefficients that our three fabricated SAW feeders could stably and continuously feed the powders.

Next, to investigate the accuracy of the powder feed weight with our fabricated SAW feeder, we modified the microcomputer program by which the SAW feeder was controlled, as shown in the timing chart of Fig. 17. Then, we measured the fed powder weights $\Delta m$ over driving 
durations $\Delta t$ of $50,100,200$, and $400 \mathrm{~ms}$. Here, for each duration, the fed powder weight was measured 10 times repeatedly. In the experiment, when the alumina and polyethylene powders were used, clogs often formed under the hole of the hopper when the SAW feeder was driven intermittently. Thus, in this paper, we describe the accuracy of the powder feed weights for copper and glass.

The relationships between the driving duration of the SAW feeder and the fed powder weight of copper and glass are shown in Figs. 18(a) and 18(b), respectively. It was found that, for both powders, the average fed powder weight $\Delta m$ linearly increased with the driving duration $\Delta t$. Moreover, it was also found that the standard deviation of the fed powder weight of copper increased with the driving duration, whereas that of the glass was almost constant. We presumed that the difference in standard deviation between the copper and glass powders was influenced by the difference in their particle size distributions (Fig. 3), i.e., the accuracy of the fed powder weight increased when powder with a narrower particle size distribution was used.

Here, from the values of the fed powder weights at the minimum driving duration of $50 \mathrm{~ms}$ as the top performances of the fabricated SAW feeders, the average weights of the copper and glass powders were $2.3 \mathrm{mg}$ (standard deviation of $0.4 \mathrm{mg}$ ) and $0.9 \mathrm{mg}$ (standard deviation of $0.2 \mathrm{mg}$ ), respectively. The result shows that, by using SAW actuators, we succeeded in feeding a few milligrams of dry powder with a particle diameter of $100 \mu \mathrm{m}$ or less, as required for custom-made pharmaceuticals and other applications, although the range of powders that can be fed is limited at present.
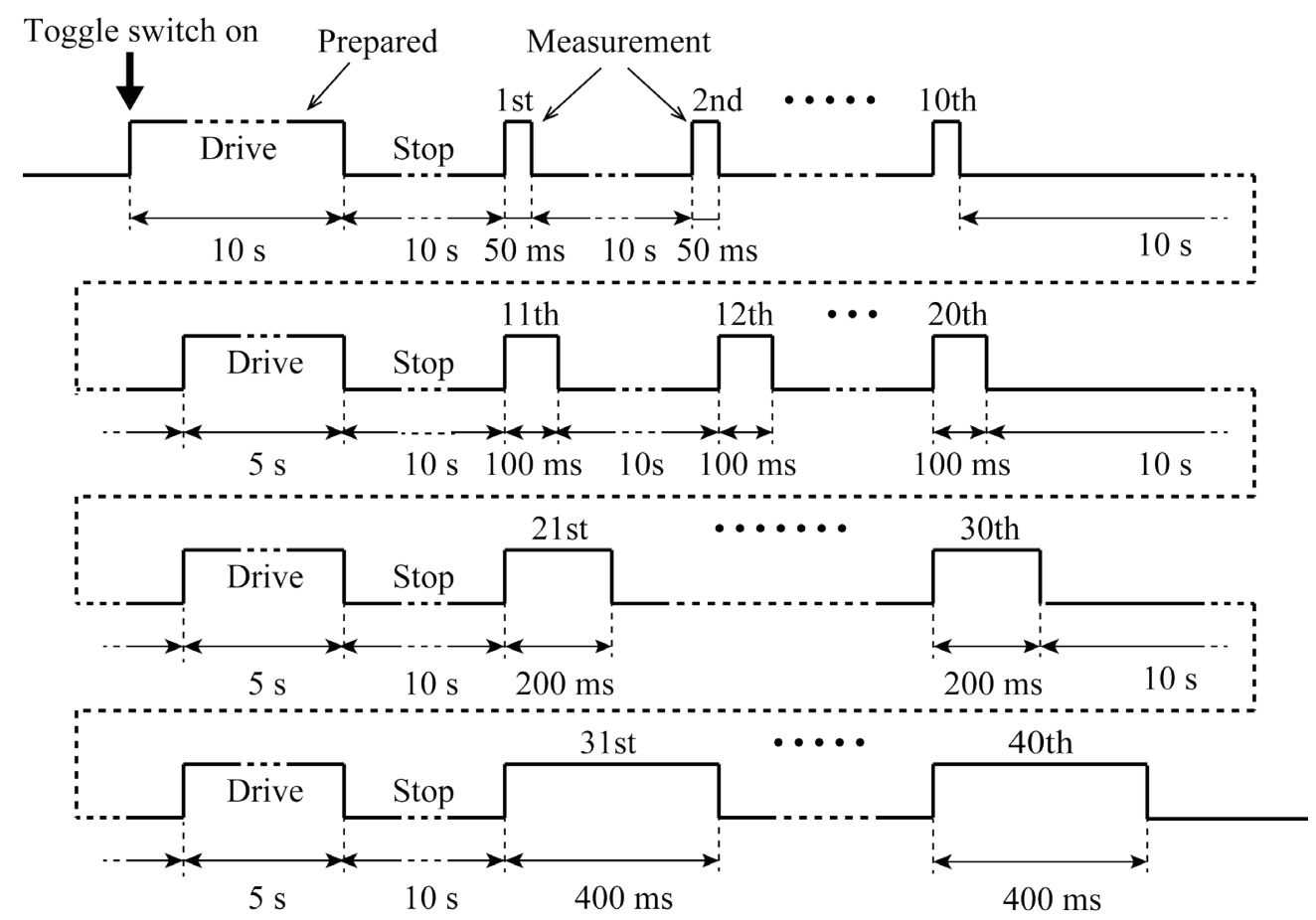

Fig. 17. Timing chart of SAW feeder operation for investigating accuracy of fed powder weight. 


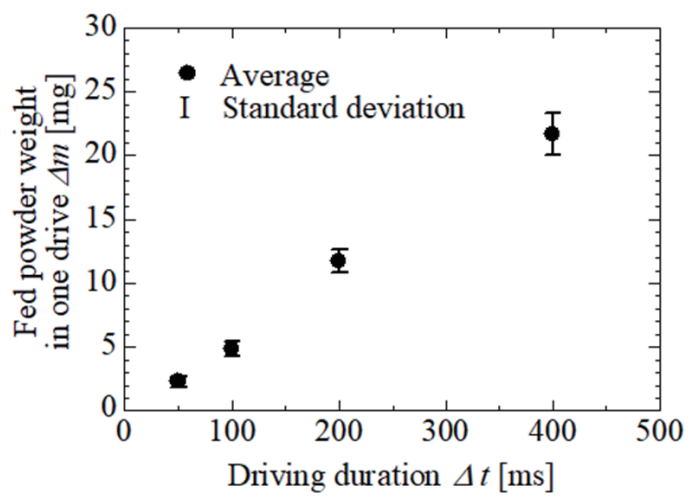

(a)

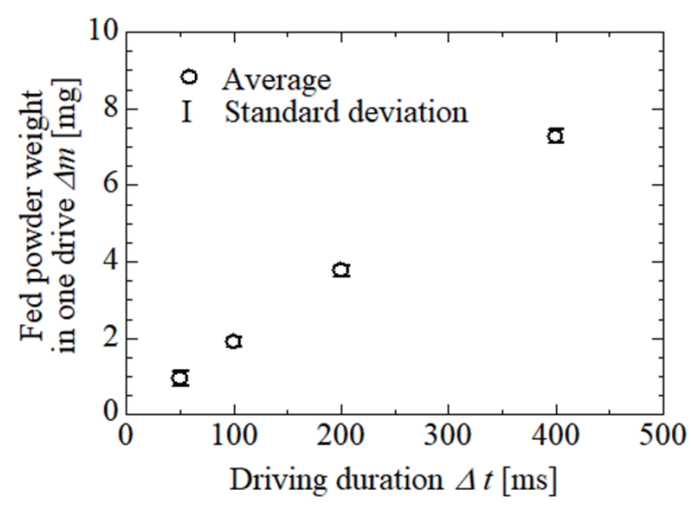

(b)

Fig. 18. Relationships between driving duration of SAW feeder and fed powder weight $\left(P_{A}=1 \mathrm{~W}\right)$. (a) Copper powder, $\theta=0^{\circ}$ and (b) glass powder, $\theta=6^{\circ}$.

\section{Conclusions}

For $\mu \mathrm{TAS}$ and other applications, there has been a need to physically handle tiny amounts of various dry powders. Therefore, we focused on a SAW actuator whose structure is very simple and investigated the different kinds of powders that could be transported by SAWs. The SAW actuators used in our experiments were fabricated by patterning an IDT on an $18 \times 78 \mathrm{~mm}^{2}$ substrate obtained by cutting a 4 -inch piezoelectric $\left(127.8^{\circ} y\right.$-rotated $x$-propagating $\left.\mathrm{LiNbO}_{3}\right)$ wafer. The IDT had a $2 \mathrm{~mm}$ stripline pitch, a $10 \mathrm{~mm}$ aperture, and 10 stripline pairs, and its central axis direction was aligned with the orientation flat direction of the wafer.

In a fundamental experiment on powder transport, we kept the SAW actuator, i.e., the SAWpropagating surface for transporting powder, horizontal. Then, to generate SAWs, we applied a high-frequency electric power of $1 \mathrm{~W}$ with a $3.2 \mathrm{MHz}$ drive frequency to the IDT. As samples for investigating whether powder could be transported or not, we used four kinds of powders with spherical copper, spherical glass, spherical polyethylene, and angular alumina particles, with particle sizes of about $100 \mu \mathrm{m}$. The results showed that only the copper powder could be transported by using SAWs.

We then closely observed the particle movement behaviors of the powders that could not be transported. It was found that, on the SAW-propagating surface, these particles did not stop at certain places but moved in various directions. This indicates that, by propagating SAWs on the surface where powders were placed, the powder particles were released from binding effects generated by static electricity and other causes. On the basis of this obtained knowledge, to transport the powders that could not be transported only by SAWs, we proposed a method that utilizes the force of gravity. Concretely, by tilting the SAW-propagating surface for transporting the powder, i.e., the SAW actuator, from a horizontal state, the force of gravity was applied to all particles in the desired direction of powder transport. Then, we experimented on powder transport by using this method. It was found that the three kinds of powders that could not be transported in the horizontal state could be transported by tilting the SAW actuator. 
Next, on the basis of the copper powder transport efficiency of $15.4 \mathrm{~mm} /(\mathrm{s} \cdot \mathrm{W})$ when the SAW actuator was kept horizontal $(\theta=0)$ and an electric power of $1 \mathrm{~W}$ was applied to it, we investigated the tilting angles $\theta$ of the other kinds of powders that gave the same transport efficiency. It was found that the tilting angles with the same transport efficiency for the glass, polyethylene, and alumina powders were $6^{\circ}, 15^{\circ}$, and $26^{\circ}$, respectively. Moreover, when the SAW actuator was tilted at these angles, the response times of the powder moved by the SAWs were very short, $50 \mathrm{~ms}$ or less. This indicates that the SAW actuator has great potential for transporting dry micropowders with a good response time.

Finally, we fabricated miniature feeders by mounting hoppers with a $0.4 \mathrm{~cm}^{3}$ capacity and guide walls on SAW actuators tilted at the above-mentioned angles and investigated their powder feed characteristics while applying an electric power of $1 \mathrm{~W}$ to the IDTs of these feeders. It was found that the feed weights per unit time of the copper, glass, and polyethylene powders were 50.1, 20.7, and $3.1 \mathrm{mg} / \mathrm{s}$, respectively. However, the alumina powder could not be fed at all by our fabricated SAW feeders because a flowability problem arose from the clogging of the alumina powder in our feeder. A similar flowability problem also occurred in the form of a rat hole when transporting the polyethylene powder. Moreover, for the copper and glass powders without flowability problems, we investigated the accuracies of the powder weight fed by our fabricated SAW feeders. It was found that, when the feeders were driven for $50 \mathrm{~ms}$, the average feed weights of the copper and glass powders were $2.3 \mathrm{mg}$ (standard deviation of $0.4 \mathrm{mg}$ ) and $0.9 \mathrm{mg}$ (standard deviation of $0.2 \mathrm{mg}$ ), respectively. This indicates our success in using SAWs to feed some small amounts (several milligrams) of some dry powders whose particle diameters were $100 \mu \mathrm{m}$ or less.

In the future, to solve the flowability problems that arose when using the polyethylene and alumina powders, we plan to improve the feeder parts of the hopper. We will develop a SAW feeder that can transport and feed various kinds of dry micropowders with high accuracy.

\section{Acknowledgments}

This research was supported by Grant-in-Aid for Scientific Research (C) No. 16K06170 from the Japan Society for the Promotion of Science (JSPS).

\section{References}

1 J. Ruzicka and E. H. Hansen: Anal. Chim. Acta 78 (1975) 145. https://doi.org/10.1016/S0003-2670(01)84761-9

2 S. C. Terry, J. H. Jerman, and J. B. Engell: IEEE Trans. Electron. Devices ED-26 (1979) 1880. https://doi. org/10.1109/T-ED.1979.19791

3 Y. Kikutani, T. Horiuchi, K. Uchiyama, H. Hisamoto. M. Tokeshi, and T. Kitamori: Lab Chip 2 (2002) 188. https://doi.org/10.1039/B208382P

4 M. Mracek and J. Wallaschek: Mater. Chem. Phys. 90 (2005) 378. https://doi.org/10.1016/ j.matchemphys.2004.09.048

5 N. Kanbe, Y. Tomikawa, and T. Takano: Jpn. J. Appl. Phys. 32 (1993) 2405. https://doi.org/10.1143/ JJAP.32.2405

6 U. Kuhne and U. Fristsching: Powder Tech. 155 (2005) 117. https://doi.org/10.1016/j.powtec.2005.05.050

7 T. Saiki, A. Tsubosaka, A. Yamaguchi, M. Suzuki, and Y. Utsumi: Adv. Powder Tech. 28 (2017) 491. https:// doi.org/10.1016/j.apt.2016.11.006

8 A. Sano, Y. Matsui, and S. Shiokawa: Jpn. J. Appl. Phys. 37 (1998) 2979. https://doi.org/10.1143/JJAP.37.2979 
9 A. Wixforth: Superlattices Microstruct. 33 (2003) 389. https://doi.org/10.1016/j.spmi.2004.02.015

10 K. Sritharan, C. J. Strobl, M. F. Schneider, and A. Wixforth: Appl. Phys. Lett. 88 (2006) 054102. https://doi. org/10.1063/1.2171482

11 L. Y. Yeo and J. R. Friend: Biomicrofluidics 3 (2009) 012002. https://doi.org/10.1063/1.3056040

12 T. Saiki, K. Okada, and Y. Utsumi: Microsyst. Technol. 16 (2010) 1589. https://doi.org/10.1007/s00542-0101044-6

13 T. Saiki, K. Okada, and Y. Utsumi: Electron. Commun. Jpn. 94 (2011) 10. https://doi.org/10.1002/ecj.10367

14 T. Saiki and Y. Utsumi: Electron. Commun. Jpn. 97 (2014) 54. https://doi.org/10.1002/ecj.11440

15 M. Kurosawa, M. Takahashi, and T. Higuchi: IEEE Trans. Ultrason. Ferroelectr. Freq. Control 43 (1996) 901. https://doi.org/10.1109/58.535493

16 Powder process.net: https://www.powderprocess.net/Powder_Flow/Arching_Bridging.html (accessed February 2021). 